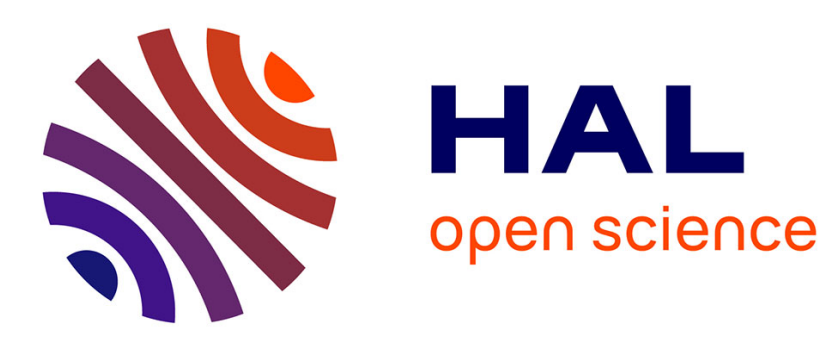

\title{
Morphological Scale-Space Operators for Images Supported on Point Clouds
}

Jesus Angulo

\section{To cite this version:}

Jesus Angulo. Morphological Scale-Space Operators for Images Supported on Point Clouds. 5th International Conference on Scale Space and Variational Methods in Computer Vision, Jun 2015, Lège-Cap Ferret, France. 10.1007/978-3-319-18461-6_7 . hal-01108141v3

HAL Id: hal-01108141

https://hal-mines-paristech.archives-ouvertes.fr/hal-01108141v3

Submitted on 17 Jan 2016

HAL is a multi-disciplinary open access archive for the deposit and dissemination of scientific research documents, whether they are published or not. The documents may come from teaching and research institutions in France or abroad, or from public or private research centers.
L'archive ouverte pluridisciplinaire HAL, est destinée au dépôt et à la diffusion de documents scientifiques de niveau recherche, publiés ou non, émanant des établissements d'enseignement et de recherche français ou étrangers, des laboratoires publics ou privés. 


\title{
Morphological Scale-Space Operators for Images Supported on Point Clouds
}

\author{
Jesús Angulo \\ MINES ParisTech, PSL-Research University, \\ CMM-Centre de Morphologie Mathématique, France \\ jesus.angulo@mines-paristech.fr
}

\begin{abstract}
The aim of this paper is to develop the theory, and to propose an algorithm, for morphological processing of images painted on point clouds, viewed as a length metric measure space $(X, d, \mu)$. In order to extend morphological operators to process point cloud supported images, one needs to define dilation and erosion as semigroup operators on $(X, d)$. That corresponds to a supremal convolution (and infimal convolution) using admissible structuring function on $(X, d)$. From a more theoretical perspective, we introduce the notion of abstract structuring functions formulated on length metric Maslov idempotent measurable spaces, which is the appropriate setting for $(X, d)$. In practice, computation of Maslov structuring function is approached by a random walks framework to estimate heat kernel on $(X, d, \mu)$, followed by the logarithmic trick.
\end{abstract}

Keywords: mathematical morphology ; point clouds image; metric measure space; idempotent measure; Hamilton-Jacobi semigroup

\section{Introduction}

With the development of 3D scanning technology it is now easy to generate 3D models from real objects, together with standard images painted on them. These discrete objects can be represented either by surfaces (typically as polygonal meshes) or by scattered point clouds. In this paper we focus exclusively on a point cloud representation. A typical case of such generalized 3D images are the point clouds obtained from range cameras, like the Kinect camera. Indeed, this is the case-study which will be used in the paper to illustrate our methods.

Let us formalize the notion of point cloud and of image on it. A point cloud $(X, d)$ is a finite set of points $X=\left\{x_{i}\right\}_{i=1}^{N}$ equipped with a distance function $d$, which corresponds typically to the Euclidean distance in the ambient space where $X$ is endowed. This set of points has been irregularly sampled from a metric space; typically $X \subset \mathbb{R}^{d}$ (for instance the $3 \mathrm{D}$ space) but $X$ can be also a sampled manifold with possible complex topological/geometric structure. We assume unknown inner product and geodesic structure of the underlying manifold. It is also important to note that $(X, d)$ is neither a graph nor a discretized 
(a)

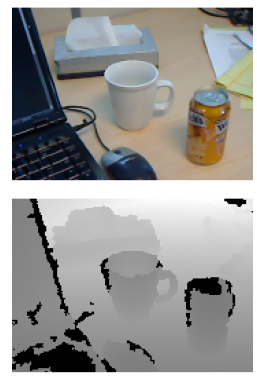

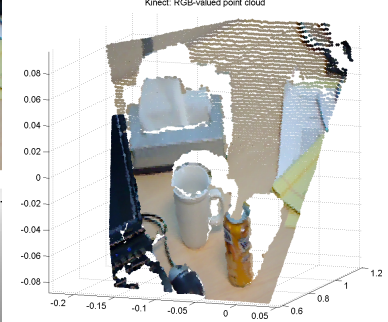

(c)

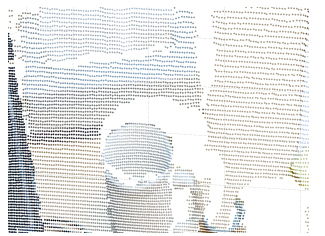

(d)

Fig. 1. Example of RGB-valued point cloud from a Kinect image: (a) $f_{\text {color }}(x)$, (b) $f_{\text {depth }}(x),\left(\right.$ c) $f\left(x_{i}\right),($ d) zoom-in of $(\mathrm{c})$.

surface with triangulations. Thus there is a lack of natural connectivity (or neighborhood relationship) between the points. A real-valued point cloud $(X, d, f)$, or image $f$ supported on a point cloud $(X, d)$, is the function $f$ which maps each point of $X$ to a valuation space, typically the extended real line for gray-scale images, i.e., $f:\left\{x_{i}\right\}_{i=1}^{N} \longrightarrow \overline{\mathbb{R}}$. Fig. 1 depicts an example of RGB-valued point cloud obtained from a Kinect image, based on the 3D reconstruction of the scene according to the $f_{\text {depth }}(x)$ image.

Point clouds processing is an active research area which have been approached using geodesic distance methods [19], laplace operator [6] or diffusion PDEs [14]. Connection between heat method on manifolds and shortest geodesic distance via the Varadhan's formula has been used in [9], which integrates the heat flow. Morphological operators have been also considered for point cloud processing [8]. However, here we are interested on processing images painted on point clouds, without modifying the point cloud itself. The problem of smoothing images painted on surfaces using the short-time Beltrami kernel was considered for instance in [23].

In this context, the aim of this paper is to develop the theory and to propose an algorithm for morphological processing of such generalized manifold images.

Mathematical morphology is a nonlinear image processing methodology based on two basic operators, dilation and erosion, which correspond respectively to the convolution in the max-plus algebra and its dual. More precisely, in Euclidean (translation invariant) mathematical morphology the pair of adjoint and dual operators, dilation (sup-convolution) $(f \oplus b)(x)$ and erosion (inf-convolution) $(f \ominus b)(x)$ of an image $f: E \subset \mathbb{R}^{n} \rightarrow \overline{\mathbb{R}}=\mathbb{R} \cup\{-\infty,+\infty\}$, are given by [21,12]:

$$
\left\{\begin{array}{l}
\delta_{b}(f)(x)=(f \oplus b)(x)=\sup _{y \in E}\{f(y)+b(y-x)\}, \\
\varepsilon_{b}(f)(x)=(f \ominus b)(x)=\inf _{y \in E}\{f(y)-b(y+x)\}
\end{array}\right.
$$

where $b: \mathbb{R}^{n} \rightarrow \overline{\mathbb{R}}$ is the structuring function which determines the effect of the operator. The structuring function plays a similar role to the kernel in classical convolution filtering. The structuring function is typically a parametric family $b_{t}(x)$, where $t>0$ is the scale parameter. In particular, the canonic 
structuring function is the parabolic shape (i.e., square of the Euclidean distance): $b_{t}(x)=-\frac{\|x\|^{2}}{2 t}$, such that the corresponding dilation and erosion are equivalent to the viscosity solution of the standard Hamilton-Jacobi PDE [17]: $u_{t}(t, x) \mp\left\|u_{x}(t, x)\right\|^{2}=0,(t, x) \in(0,+\infty) \times E ; u(0, x)=f(x), x \in E$. Theory of morphological filtering is based on opening and closing operators, obtained respectively by composition product of erosion-dilation and dilation-erosion. Opening (resp. closing) is increasing, idempotent and anti-extensive (resp. extensive). Evolved filters are obtained by composition of openings and closings [21,12]. Morphological operators are classically defined for images supported on Euclidean spaces. A recent work has introduced mathematical morphology for real valued images whose support space is a Riemannian manifold [5].

In order to extend morphological operators to process point cloud supported images, one needs to define dilation and erosion as semigroup operators in framework adapted to point clouds $(X, d)$. That corresponds to a supremal convolution (and infimal convolution) using admissible structuring function on $(X, d)$. These operators are formulated in Section 3. In a more theoretical perspective, we start in Section 2 by introducing the notion of abstract structuring functions formulated on length metric Maslov idempotent measurable spaces, which is the appropriate setting for $(X, d)$. In practice, computation of Maslov structuring function will be approached by a random walk framework to estimate heat kernel on a metric measure space $(X, d, \mu)$, followed by the logarithmic trick, as shown in Section 4. Some preliminary examples of morphological processing RGB-valued point cloud images are also given in Section 4.

\section{From abstract heat semigroups to Maslov structuring functions}

Before introducing the morphological framework, let us review the theory of abstract heat kernels on a metric measure space, which is based on recent works by Grigor'yan and co-workers [10,11].

\section{$2.1 \quad$ Heat kernel on metric measure spaces}

Let $(M, d, \mu)$ be a locally compact, separable metric space, endowed with a Radon measure $\mu$. Then a family $\left\{p_{t}\right\}_{t \geq 0}$ of measurable functions $p_{t}(x, y)$ on $M \times M$ is called a heat kernel is the following conditions are almost surely satisfied $\forall x, y \in M$ and $t, s>0$ : i) Positivity: $p_{t}(x, y) \geq 0$; ii) Total mass inequality: $\int_{M} p_{t}(x, y) d \mu(y) \leq 1$, iii) Symmetry: $p_{t}(x, y)=p_{t}(y, x)$, iv) Semigroup property $\left.p_{s+t}(x, y)=\int_{M} p_{s}(x, z) p_{t}(z, y) d \mu(z), \mathrm{v}\right)$ Approximation to identity: For any $f \in$ $L^{2}(M, \mu) \int_{M} p_{t}(x, y) f(y) d \mu(y) \longrightarrow{ }^{L^{2}} f(x)$ as $t \rightarrow 0^{+}$. In addition, the heat kernel is called stochastically complete (conservative) when $\int_{M} p_{t}(x, y) d \mu(y)=1$.

Given a measurable function $f$ on $M$, any heat kernel gives rise to the family of operators $\left\{P_{t}\right\}_{t \geq 0}$, called the heat semigroup, where $P_{0}=$ id and $P_{t}$ for $t>0$ is defined by

$$
P_{t} f(x)=\int_{M} p_{t}(x, y) f(y) d \mu(y)
$$


such that $\left\{P_{t}\right\}_{t \geq 0}$ is continuous, symmetric and a Markovian semigroup $P_{t} P_{s}=$ $P_{s+t}$.

Heat kernels and heat semigroups arise naturally from Markov processes. Let $\left(\left\{X_{t}\right\}_{t \geq 0} ;\left\{\mathbb{P}_{x}\right\}_{x \in M}\right)$ be a Markov process on $M$, that is reversible with respect to measure $\mu$. Assume that it has the transition density $p_{t}(x, y)$, i.e., a function such that $\forall x \in \mathcal{M}, t>0$, and all Borel sets $A \subset M$, one has $\mathbb{P}_{x}\left(X_{t} \in A\right)=$ $\int_{M} p_{t}(x, y) d \mu(y)$.

If $M$ is a smooth connected compact Riemannian manifold such that $\triangle_{\mu}$ is the Laplace-Beltrami operator on $M$, where $\mu$ is the Riemannian measure, it is well known that starting from the heat equation $\frac{\partial u}{\partial t}=\triangle_{\mu} u$, we have that, for any $y \in M$, the function $(t, x) \mapsto p_{t}(x, y)$ is the smallest positive fundamental solution of the heat equation with a source at $y$, i.e., if $u(x, 0)=u_{0}(x)$, then $u(x, t)=\int_{M} p_{t}(x, y) u_{0}(y) d \mu(y)$. In addition, let $\left\{\varphi_{k}\right\}_{k=0}^{+\infty}$ be an orthonormal basis of eigenfunctions of Laplace-Beltrami operator $-\triangle_{\mu}$, with eigenvalues $0=\lambda_{0}<\lambda_{1} \leq \lambda_{2} \leq \cdots$, then the heat kernel is determined by $p_{t}(x, y)=$ $\sum_{k=0}^{+\infty} e^{-\lambda_{k} t} \varphi_{k}(x) \varphi_{k}(y)$.

As mentioned in the introduction, all these different viewpoints on heat kernel theory have been considered in the image processing state-of-the-art dealing with meshes and point clouds.

The explicit expression of the heat kernel in the Euclidean space $(M, d, \mu)=$ $\mathbb{R}^{n}$ is just the Gaussian kernel. In the case of a Riemannian manifold $(M, \mu)$, only estimates are available, which typically depends on the geodesic distance in $M$, see $[10,11]$. Similarly, in the case of a metric measure space $(M, d, \mu)$, the sub-Gaussian estimate has the following form (assuming a walk dimension equals to 2) $[10,11]$ :

$$
p_{t}(x, y) \asymp \frac{C}{V\left(x, t^{1 / 2}\right)} \exp \left(-c \frac{d^{2}(x, y)}{t}\right)
$$

where $d(x, y)$ is a metric, $V(x, r)$ is the volume function of a metric ball on the space and $C$ and $c$ are positive constants.

\subsection{Structuring functions on length metric Maslov measure spaces}

We need the counterpart of this abstract theory in the context max-plus mathematics (also known as idempotent analysis [18,15]). More precisely, the max-plus equivalent of the heat kernel will be here named structuring functions on a length metric Maslov measure space.

Length metric Maslov measure space. Theoretical foundations of Maslov idempotent measure theory $[20,1]$ are based on replacing in the structural axioms of probability theory the role of the classical semiring $\mathrm{S}_{(+, \times)}=\left(\mathbb{R}_{+},+, \times, 0,1, \leq\right)$ of positive real numbers by the idempotent semiring:

$\mathrm{S}_{(\max ,+)}=(\overline{\mathbb{R}}, \max ,+,-\infty, 0, \leq)$. In this context, a change of the measure involves a consistent counterpart to the standard probability theory. 
Let $(X, d)$ be a (Hausdorff topological) metric space and let $\mathfrak{m}$ be a Maslov idempotent measure on $X$, i.e., mapping from $X$ in the max-plus semiring such that for every function $f: X \rightarrow \overline{\mathbb{R}}$, we have $A \subset X$,

$$
\mathfrak{m}_{f}(A)=\sup _{x \in A} f(x) .
$$

In addition, we should assume that $(X, d)$ is a length space. We remind that a length space is a metric space such that for any pair of points, their distance is the length of the shortest path between the two points. The triple $(X, d, \mathfrak{m})$ is called a length metric Maslov measure space.

Maslov structuring function. A family $\left\{\mathfrak{b}_{t}\right\}_{t>0}$ of Maslov idempotent measurable functions $\mathfrak{b}_{t}: X \times X \rightarrow \overline{\mathbb{R}}$ is called for us a Maslov structuring function in $(X, d, \mathfrak{m})$ if the following conditions are satisfied $\forall x, y \in X$ and all $t, s>0$

- Nonpositivity and total mass inequality:

$$
\mathfrak{m}\left(\mathfrak{b}_{t}(x, \cdot)\right)=\sup _{y \in X} \mathfrak{b}_{t}(x, y) \leq 0 \Leftrightarrow \mathfrak{b}_{t}(x, y) \leq 0 .
$$

- Completeness (or conservative):

$$
\mathfrak{m}\left(\mathfrak{b}_{t}(x, x)\right)=0 \Leftrightarrow \mathfrak{b}_{t}(x, x)=0
$$

- Symmetry: $\mathfrak{b}_{t}(x, y)=\mathfrak{b}_{t}(y, x)$.

- Semi-group property:

$$
\mathfrak{b}_{t+s}(x, y)=\sup _{z \in X}\left\{\mathfrak{b}_{t}(x, z)+\mathfrak{b}_{s}(z, y)\right\}
$$

- Approximation to identity:

$$
\sup _{y \in X}\left\{f(y)+\mathfrak{b}_{t}(x, y)\right\} \longrightarrow f(x) \text { as } t \rightarrow 0^{+} .
$$

Logarithmic trick: from heat kernel to Maslov structuring function. Our approach is based on the so-called logarithmic connection between linear and morphological system theory [7]. The theory of this connection between the usual convolution and the convolution in max-plus mathematics is based on the Cramer transform [1], which is defined as the Legendre transform of the logarithm of the Laplace transform. In the particular case of a canonic Gaussian kernel of type: $1 / \sqrt{2 \pi \sigma^{2}} e^{-x^{2} /\left(2 \sigma^{2}\right)}$, it is well known that the Laplace transform is $e^{\left(\sigma^{2} s^{2}\right) / 2}$ and the final Cramer transform is $-x^{2} /\left(2 \sigma^{2}\right)$. In summary the Cramer transform of the Gaussian kernel is equal to quadratic structuring function.

Similarly, we can easily show that, up to a normalizing constant, the logarithm of the heat kernel $p_{t}(x, y)$ in a metric measure space $(X, d, \mu)$ is a Maslov structuring function $\mathfrak{b}_{t}(x, y)$ in the counterpart idempotent metric measure space. 
Let us consider for instance the case of the sub-Gaussian estimate of the heat kernel (2). First, a normalization version $\tilde{p}_{t}(x, y)$ is required such that $\tilde{p}_{t}(x, x)=1$, i.e., $\tilde{p}_{t}(x, y)=V\left(x, t^{1 / 2}\right) / C p_{t}(x, y)$. Then, the corresponding Maslov structuring function is defined as

$$
\mathfrak{b}_{t}(x, y)=\log \tilde{p}_{t}(x, y) \asymp-c \frac{d^{2}(x, y)}{t},
$$

and, without loss of generality, we fix $c=1 / 2$. The nonpositivity, total mass inequality, completeness, symmetric and approximation to the identity are obvious. In order to prove the semigroup property, we need to assume that $d(x, y)$ is a metric distance in a length space such that [16]

$$
\begin{aligned}
\mathfrak{b}_{t+s}(x, y) & \left.=\sup _{z \in X}\left\{\mathfrak{b}_{t}(x, z)+\mathfrak{b}_{s}(z, y)\right\}=\sup _{z \in X}\left\{-\frac{d^{2}(x, z)}{2 t}-\frac{d^{2}(z, y)}{2 s}\right\}\right\} \\
& \left.=-\inf _{z \in X}\left\{\frac{d^{2}(x, z)}{2 t}+\frac{d^{2}(z, y)}{2 s}\right\}\right\}=-\frac{d^{2}(x, y)}{2(t+s)}
\end{aligned}
$$

The last equality in length spaces comes from $d^{2}(x, y) /(2(t+s)) \leq \inf _{z \in Z}$ $\left[d^{2}(x, z) /(2 t)+d^{2}(z, y) /(2 t)\right]$ (by triangle inequality) by choosing a minimal geodesic between $x$ and $y$, and a point $z$ on this geodesic with $d(x, z)=t /(t+$ $s) d(x, y)$.

\section{Morphological scale-space operators on metric Maslov-measurable space}

Let us consider a metric Maslov-measurable space $(X, d, \mathfrak{m})$ and a given function $f: X \rightarrow \overline{\mathbb{R}}$. Once an admissible structuring function $\left\{\mathfrak{b}_{t}\right\}_{t>0}$ has been defined, the max-plus semigroups $D_{t} f(x)$ and $E_{t} f(x)$ are given by

$$
\begin{aligned}
& D_{t} f(x)=\sup _{y \in X}\left\{f(y)+\mathfrak{b}_{t}(x, y)\right\}, \\
& E_{t} f(x)=\inf _{y \in X}\left\{f(y)-\mathfrak{b}_{t}(y, x)\right\} .
\end{aligned}
$$

which, in the context of mathematical morphology, correspond respectively to the multi-scale dilation $\delta_{\mathfrak{b}_{t}}(f)(x)=D_{t} f(x)$ and erosion $\varepsilon_{\mathfrak{b}_{t}}(f)(x)=E_{t} f(x)$ of function $f$ by structuring function $\mathfrak{b}_{t}$. By the way, we note that by the symmetry, one has $\mathfrak{b}_{t}(x, y)=\mathfrak{b}_{t}(y, x)$. In max-plus mathematics on Hilbert spaces, dilation and erosion are known as the Hopf-Lax-Oleinik semigroups. By considering for instance the sub-gaussian estimate as Maslov structuring function (3), the obtained semigroups in $(X, d, \mathfrak{m}): D_{t} f(x)=\sup _{y \in X}\left\{f(y)-\frac{d^{2}(x, y)}{2 t}\right\}$ and $E_{t} f(x)=\inf _{y \in X}\left\{f(y)+\frac{d^{2}(x, y)}{2 t}\right\}$, are equal to the so-called Hamilton-Jacobi semigroup on length spaces, introduced by Lott and Villani in [16] and extendedly considered more recently by Ambrosio et al. in [3] for the study of Ricci curvature bounds in metric spaces. 


\subsection{Properties of semigroups $\delta_{\mathfrak{b}_{t}}$ and $\varepsilon_{\mathfrak{b}_{t}}$}

The following properties can be easily proved using the properties of a Maslov structuring function.

1. (Adjunction) For any two real-valued functions $f$ and $g$ on $(X, d, \mathfrak{m})$, the pair $\left(\varepsilon_{\mathfrak{b}_{t}}, \delta_{\mathfrak{b}_{t}}\right)$ forms an adjunction, i.e., $\forall x \in X$

$$
\delta_{\mathfrak{b}_{t}}(f)(x) \leq g(x) \Leftrightarrow f(x) \leq \varepsilon_{\mathfrak{b}_{t}}(g)(x) .
$$

2. (Duality by involution) For any function $f(x)$ and $\forall x \in X$, one has $\delta_{\mathfrak{b}_{t}}(f)(x)=$ $-\varepsilon_{\mathfrak{b}_{t}}(-f)(x)$.

3. (Increaseness) If $f(x) \leq g(x), \forall x \in X$, then $\delta_{\mathfrak{b}_{t}}(f)(x) \leq \delta_{\mathfrak{b}_{t}}(g)(x)$ and $\varepsilon_{\mathfrak{b}_{t}}(f)(x) \leq \varepsilon_{\mathfrak{b}_{t}}(g)(x), \forall x \in X$ and $\forall t>0$.

4. (Extensivity and anti-extensivity) $\delta_{\mathfrak{b}_{t}}(f)(x) \geq f(x)$ and $\varepsilon_{\mathfrak{b}_{t}}(f)(x) \leq f(x)$, $\forall x \in X$ and $\forall t>0$.

5. (Ordering property) If $0<s<t$ then $\varepsilon_{\mathfrak{b}_{t}}(f)(x) \leq \varepsilon_{\mathfrak{b}_{s}}(f)(x) \leq f(x)$ $\delta_{\mathfrak{b}_{s}}(f)(x) \leq \delta_{\mathfrak{b}_{t}}(f)(x), \forall x \in X$.

6. (Scale-space) For any function $f$ and $\forall x \in X$, and for all pair of scales $s>0$ and $t>0$, we have the Maslovian semigroup:

$$
\begin{aligned}
& \delta_{\mathfrak{b}_{t}}\left(\delta_{\mathfrak{b}_{s}}(f)\right)(x)=\delta_{\mathfrak{b}_{s+t}}(f)(x), \\
& \varepsilon_{\mathfrak{b}_{t}}\left(\varepsilon_{\mathfrak{b}_{s}}(f)\right)(x)=\varepsilon_{\mathfrak{b}_{s+t}}(f)(x) .
\end{aligned}
$$

In addition, the semigroups operators $\delta_{\mathfrak{b}_{t}}(f)$ and $\varepsilon_{\mathfrak{b}_{t}}(f)$ are continuous in $t$.

We note that these properties are a natural generalization of some well known properties of morphological scale-spaces [13,17].

\subsection{Multi-scale openings and closings and other derived operators}

From these two basic well formalized operators, all the morphological filtering theory is generalized to images on $(X, d, \mathfrak{m})$. In particular, as a consequence of the classic theory of morphological operators [21,12], the composition products of the adjoint operators $\left(\varepsilon_{\mathfrak{b}_{t}}, \delta_{\mathfrak{b}_{t}}\right)$ lead to the multi-scale opening and closing:

$$
\begin{aligned}
\gamma_{\mathfrak{b}_{t}}(f)(x) & =\delta_{\mathfrak{b}_{t}} \circ \varepsilon_{\mathfrak{b}_{t}}(f)(x)=\sup _{z \in X} \inf _{y \in X}\left\{f(y)-\mathfrak{b}_{t}(y, z)+\mathfrak{b}_{t}(z, x)\right\}, \\
\varphi_{\mathfrak{b}_{t}}(f)(x) & =\varepsilon_{\mathfrak{b}_{t}} \circ \delta_{\mathfrak{b}_{t}}(f)(x)=\inf _{z \in X} \sup _{y \in X}\left\{f(y)+\mathfrak{b}_{t}(z, y)-\mathfrak{b}_{t}(x, z)\right\} .
\end{aligned}
$$

Having the opening and closing, all the other morphological filters defined by composition of them are easily obtained, such as the alternate sequential filters. It is also possible to extend the geodesic dilation [12], denoted by $\delta_{\mathfrak{b}_{t}}^{g e o}(m ; f)$, of a marker function $m$ constrained by the reference function $f$, defined as $\delta_{\mathfrak{b}_{t}}^{g e o}(m ; f)(x)=\delta_{\mathfrak{b}_{t}}(m)(x) \wedge f(x)$ and $t$ being a small scale. The iteration of this operator until converge is called geodesic reconstruction [12]. This is an extremely useful operator which, based for instance on an opening $m=\gamma_{\mathfrak{b}_{t}}(f)$ as marker, removes the bright objects and leaving intact the contours of dark structures. 


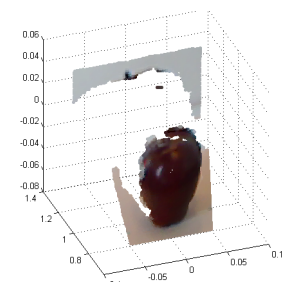

(a)

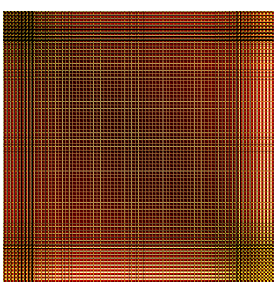

(b)

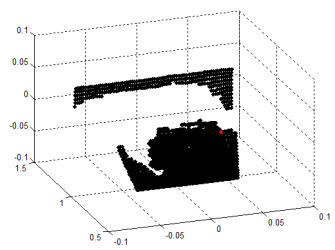

(d)

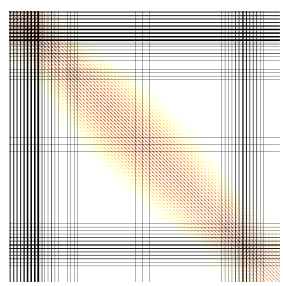

(c)

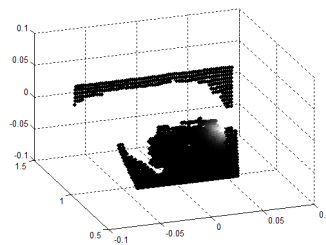

(e)

Fig. 2. Heat kernel at a given point: (a) RGB-valued point cloud $f\left(x_{i}\right)$ of $N$ points; (b) matrix of all-pairs of Euclidean distances $d^{\text {Euclid }}\left(x_{i}, x_{j}\right), 1 \leq i \leq N, 1 \leq j \leq N$; (c) matrix of all-pairs of heat distances $d_{T}^{\text {Heat }}\left(x_{i}, x_{j}\right), 1 \leq i \leq N, 1 \leq j \leq N$; (d) in red, a given point $x_{k}$, (e) heat kernel $p_{t}\left(x_{k}, x_{i}\right)$ at this point $x_{k}$.

\section{Application to processing images supported on point clouds}

\subsection{Computing Maslov structuring functions on point clouds}

According to the theory discussed above, to compute a Maslov structuring function on a point cloud is equivalent to compute the heat kernel followed by the logarithmic trick. In the case of point clouds, like those of the Kinect camera, the initial ambient space embedding $\left(X, d^{\text {Euclid }}\right)$ is not straightforwardly useful for image processing since the intrinsic geometry of the $3 \mathrm{D}$ objects is not taken into account by $d^{\text {Euclid }}(x, y)$. Local geometry of the underlaying sampled manifold could be approximated by means of manifold learning techniques (e.g., ISOMAP). That is basically computation of geodesic distances which are then plugged into the heat kernel. Another possibility involves to transform the point cloud into a triangular mesh surface. Heat kernel can be obtained by numerical computation of eigenfunctions of Laplace-Beltrami operator.

We consider here an alternative approach based on random walk paradigm, which does not need a mesh nor an explicit graph. Hence, heat diffusion can be modeled as a large collection of hot particles taking random walks on space $X$ starting at $x_{i} \in X$ : any particle that reaches a distance point $y$ after a small time has had little time to deviate from the shortest possible path. Probabilities of random walks of length $p$ can be computed by looking at the power $p$ of the transition probability matrix of the stationary Markov chain. A similar approach 
was used in [4], to formulate the notion of stochastic morphological filtering on Euclidean images.

In practice, the algorithm is based on the following steps.

1. Euclidean distance matrix. Given a point cloud $\left(X, d^{\text {Euclid }}\right)$, compute the matrix of all Euclidean distances between pairs of points of $X: D(i, j)=$ $d^{\text {Euclid }}\left(x_{i}, x_{j}\right), \forall x_{i}, x_{j} \in X$. This matrix can be made sparse by hard thresholding at $\epsilon$ (this is equivalent to so-called $\epsilon$-graph).

2. From Euclidean distance matrix to stochastic matrix. Transform distances of each point $i$ to the others (column $i$ of matrix $D$ ) into transition probabilities using Boltzmann-Gibbs distribution depending on temperature $T: P_{T}(i, j)=$ $Z_{i}^{-1} e^{-D(i, j)^{2} / T}, Z_{i}=\sum_{j} e^{-D(i, j)^{2} / T}$.

3. Probability of going from point $i$ to $j$ after $p$ steps: Compute power to $p$ of stochastic matrix $P_{T}$. Each $i$ column of the corresponding matrix can be interpreted as the approximation to the heat kernel at point $i, k_{T}^{\text {Heat }}\left(x_{i}, x_{j}\right)$, on the underlying manifold when $p \rightarrow+\infty$.

4. Maslov structuring function. Compute the scaled logarithm of the heat kernel: $\mathfrak{b}_{t}\left(x_{i}, x_{j}\right)=\frac{\log \left(k_{T}^{\text {Heat }}\left(x_{i}, x_{j}\right)\right)}{2 t}$.

Fig. 2 illustrates the approach for a RGB-valued point cloud image $f\left(x_{i}\right)$ of $N$ points. Including the shape of the heat kernel at a given point. Structure of the matrix of Euclidean distances with the respect to that of the "heat distances", obtained as $d_{T}^{\text {Heat }}\left(x_{i}, x_{j}\right)=\sqrt{-2 T \log k_{T}^{\text {Heat }}\left(x_{i}, x_{j}\right)}$, are compared too. We note that this distance can be considered as an approximation based on the wellknown Varadhan's formula which link the short time heat kernel to the geodesic distance, i.e.,

$$
d^{\text {Geodesic }}(x, y)=\lim _{t \rightarrow 0} \sqrt{-2 t \log p_{t}(x, y)} .
$$

\subsection{Examples of morphological processing}

Fig. 3 depicts some examples of morphological processing of a RGB-valued point cloud $f\left(x_{i}\right)$, using the multi-scale structuring functions $\mathfrak{b}_{t}\left(x_{i}, x_{j}\right)$ obtained by the random walk paradigm. We first note that each color component is processed separately; but using the same structuring function for the three components. The effects of morphological operators are as expected: erosion $\varepsilon_{\mathfrak{b}_{t}}(f)\left(x_{i}\right)$ in Fig. 3(b) to (f), (resp. dilation $\delta_{\mathfrak{b}_{t}}(f)\left(x_{i}\right)$ in $(\mathrm{g})$ ) enlarges dark structures and reduces bright ones (resp. enlarges bright zones and reduces dark areas). Opening $\gamma_{\mathfrak{b}_{t}}(f)\left(x_{i}\right)$, in (h) removes bright structures at the corresponding scale $t$, without modifying the dark ones. The difference between the original image $f\left(x_{i}\right)$ and the opening, given in (i), shows the removed bright zones.

Considering for instance the erosion $\varepsilon_{\mathfrak{b}_{t}}(f)\left(x_{i}\right)$ at a given scale $t=1$, it is compared, on the one hand, the effect of the temperature parameter $T$. As usual in such probabilistic framework, for very low temperatures, e.g. $T=0.05$ as in (b), the estimated heat kernel is too tightly adjusted to the local shape of the point cloud. On the contrary, with high temperatures, e.g. $T=0.4$ in (c), the 


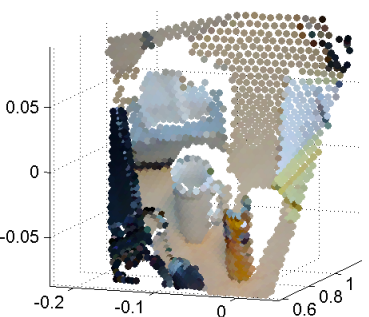

(a)

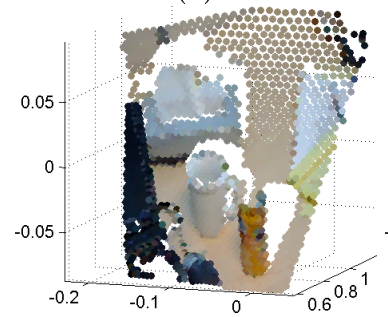

(d)

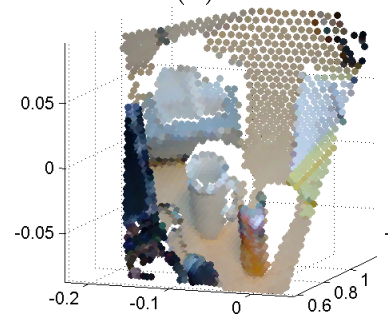

(g)

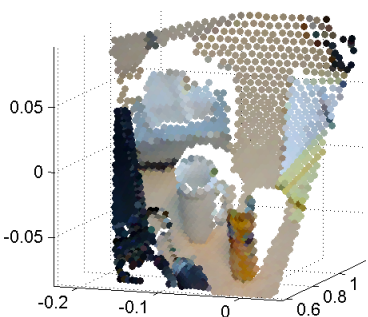

(b)

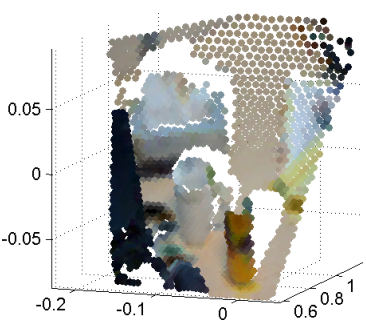

(c)

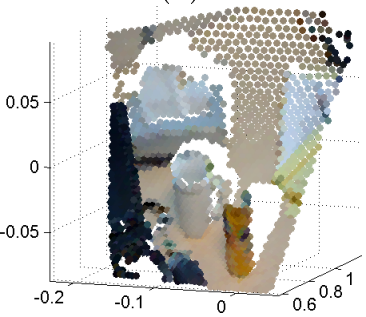

(e)

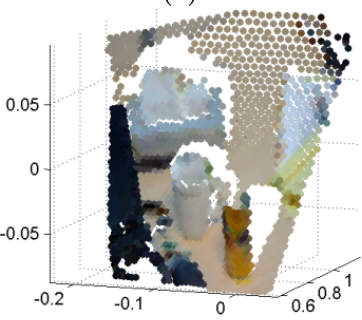

(f)

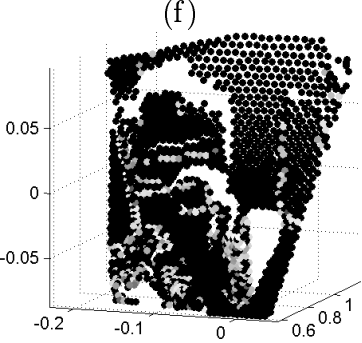

(i)

Fig. 3. Morphological processing a RGB-valued point cloud: (a) original image $f\left(x_{i}\right)$. Erosion $\varepsilon_{\mathfrak{b}_{t}}(f)\left(x_{i}\right)$ with (b) $t=1, T=0.05$, (c) $t=1, T=0.4$, (d) $t=0.5, T=0.1$, (e) $t=1, T=0.1$, (f) $t=2, T=0.1$. (g) Dilation $\delta_{\mathfrak{b}_{t}}(f)\left(x_{i}\right)$ with $t=1, T=0.1$. (h) opening $\gamma_{\mathfrak{b}_{t}}(f)\left(x_{i}\right)$ and (i) top-hat transform $f\left(x_{i}\right)-\gamma_{\mathfrak{b}_{t}}(f)\left(x_{i}\right)$ with $t=1, T=0.1$.

geometry of the point cloud is not captured by the heat kernel. For the current example, $T=0.1$ seems an appropriate choice. Then, by fixing $T=0.1$, we observe that by modifying the scale, $t=0.5(\mathrm{~d}), t=1$ (e) and $t=2$ (f), a scale-space representation is obtained.

Other examples of morphological transforms on the same image are included in Fig. 4. See legend of the figure for details.

\section{$5 \quad$ Perspectives}

We have formulated the abstract theory of morphological semi-groups on length metric Maslov-measurable spaces. These operators can be used for processing images on valued point clouds by heat kernel embedding. Typical applications 


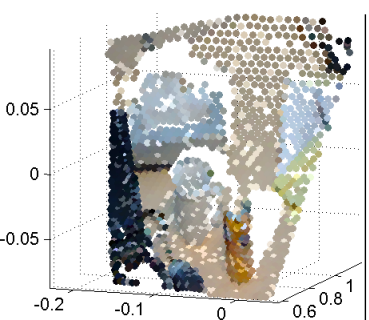

(a)

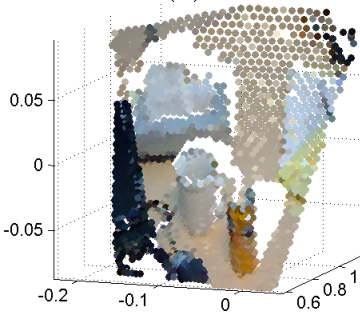

(d)

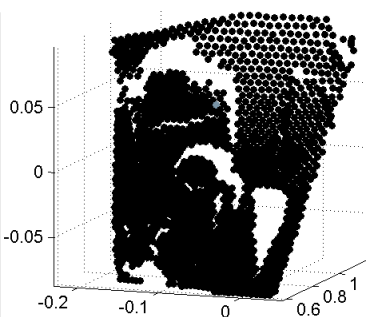

(b)

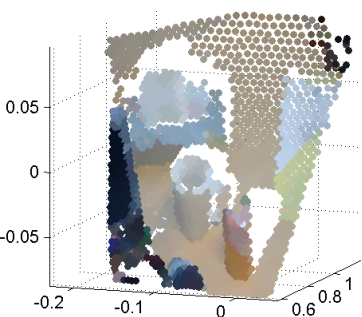

(c)

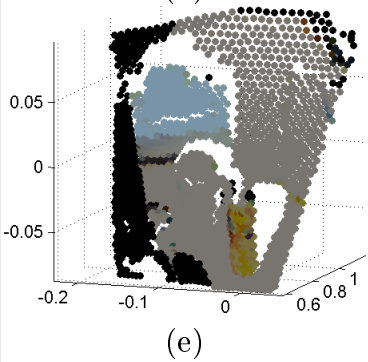

(e)

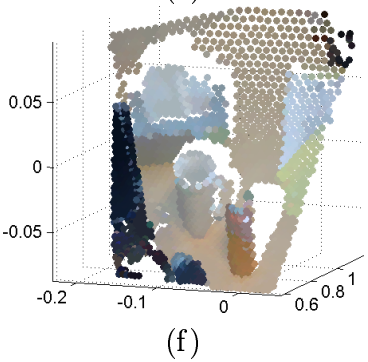

Fig. 4. Morphological processing a RGB-valued point cloud (original image in 3(a)). Corrupted image $\tilde{f}\left(x_{i}\right)$ by white impulse noise in (a) and restored image by opening $\gamma_{\mathfrak{b}_{t}}(\tilde{f})\left(x_{i}\right)$ in $(\mathrm{d})$. In (e), geodesic reconstruction using a single-pixel marker (blue point in (b)): we note that all brighter objects than the marker zones are removed. Closing $\varphi_{\mathfrak{b}_{t}}(f)\left(x_{i}\right)$ in (e), which removes dark areas, and corresponding dual geodesic reconstruction which perfectly restores contour of bright objets.

are: geometric object extraction, non-linear scale-space representation and decomposition, regularization, etc.

Our estimation of the heat kernel, based on random walks, is not very precise. A better approximation of underlying geodesic distances can be obtained by a local tangent space approximation and local mesh construction, by introducing the measure of Voronoi volumes, etc. However for the purpose of computing the Maslov structuring function on the point cloud, the random walk estimation can be considered as satisfactory.

In metric measure spaces, the heat kernel can be also approximated using Laplace-Beltrami eigenfunctions. For the need of morphological operators, the equivalent paradigm involves harmonic functions in max-plus algebra and eigenfunctions of Hopf-Lax-Oleinik semigroups. The theory is known in the Euclidean case (also some results in graph optimization) [22,2]. This point will be explored in ongoing research.

\section{References}

1. M. Akian. Densities of idempotent measures and large deviations. Trans. of the American Mathematical Society, Vol. 351, No. 11, 4515-4543, 1999. 
2. M. Akian, S. Gaubert, C. Walsh. The Max-Plus Martin boundary. Documenta Mathematica, 14: 195-240, 2009.

3. L. Ambrosio, N. Gigli, G. Savaré. Calculus and heat flow in metric measure spaces and applications to spaces with Ricci bounds from below. Inventiones mathematicae, 195(2):289-391, 2014.

4. J. Angulo, S. Velasco-Forero. Stochastic Morphological Filtering and BellmanMaslov Chains. In Proc. of ISMM'13 (11th International Symposium on Mathematical Morphology), LNCS 7883, Springer-Verlag, p. 171-182, 2013.

5. J. Angulo, S. Velasco-Forero. Riemannian Mathematical Morphology. Pattern Recognition Letters, 47:93-101, 2014.

6. M. Belkin, J. Sun, and Y. Wang. Constructing laplace operator from point clouds in $R^{d}$. In Proc. of ACM Symp. on Discrete Algorithms, 1031-1040, 2009.

7. B. Burgeth, J. Weickert. An Explanation for the Logarithmic Connection between Linear and Morphological System Theory. International Journal of Computer Vision, 64(2-3):157-169, 2005.

8. S. Calderon, T. Boubekeur. Point Morphology. ACM Transactions on Graphics (Proc. SIGGRAPH 2014), 2014.

9. K. Crane, C. Weischedel, M. Wardetzky. Geodesics in Heat: A New Approach to Computing Distance Based on Heat Flow. ACM Trans. Graph. 32(5), 2013

10. A. Grigor'yan. Heat Kernel and Analysis on Manifolds. American Mathematical Society, 2012.

11. A. Grigor'yan, J. Hu, K.-S. Lau. Heat kernels on metric measure spaces. In Geometry and Analysis on Fractals, Springer Proceedings in Mathematics $\mathcal{G}$ Statistics, $88: 147-208,2014$.

12. H.J.A.M. Heijmans. Morphological image operators. Academic Press, Boston, 1994.

13. P.T. Jackway, M. Deriche. Scale-Space Properties of the Multiscale Morphological Dilation-Erosion. IEEE Trans. Pattern Anal. Mach. Intell., 18(1): 38-51, 1996.

14. J. Liang, H. Zhao. Methods and Algorithms for Scientific Computing Solving Partial Differential Equations on Point Clouds. SIAM J. Sci. Comput., 35(3): A1461A1486, 2013.

15. G.L. Litvinov, V.P. Maslov, G.B. Shpiz. Idempotent Functional Analysis: An Algebraic Approach. Mathematical Notes, 69(5-6): 696-729, 2001.

16. J. Lott, C. Villani. Hamilton-Jacobi semigroup on length spaces and applications. Journal de Math. Pures et Appliquées, 88: 219-229, 2007.

17. P. Maragos. Differential morphology and image processing. IEEE Transactions on Image Processing, 5(1): 922-937, 1996.

18. V. Maslov. Méthodes opératorielles. Editions Mir, 1987.

19. F. Mémoli, F., G. Sapiro. A theoretical and computational framework for isometry invariant recognition of point cloud data. Foundations of Computational Mathematics, 5(3):313-347, 2005.

20. P. Del Moral. Maslov Optimization Theory: Optimality versus Randomness. In (Kolokoltsov and Maslov, Eds.) Idempotency Analysis and its Applications, Kluwer Publishers, 1997.

21. J. Serra. Image Analysis and Mathematical Morphology. Vol II: Theoretical Advances, Academic Press, London, 1988.

22. A. N. Sobolevskii. Aubry-Mather theory and idempotent eigenfunctions of Bellman operator. Commun. Contemp. Math., 1:517-533 (1999).

23. A. Spira, R. Kimmel, N. Sochen. A short-time Beltrami kernel for smoothing images and manifolds. IEEE Trans. on Image Processing, 16(6):1628-1636, 2007. 